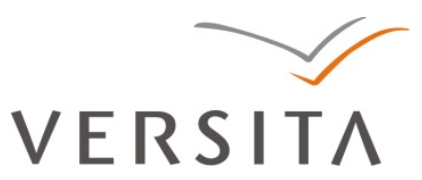

BULGARIAN ACADEMY OF SCIENCES

CYBERNETICS AND INFORMATION TECHNOLOGIES • Volume 13, No 4

Sofia $\bullet 2013$

Print ISSN: 1311-9702; Online ISSN: 1314-4081

DOI: $10.2478 /$ cait-2013-0055

\title{
Mechatronic Approaches for Functional Structural Synthesis of Mechanical Systems of Industrial Robots
}

\author{
Vitan Galabov, Vencislav Slavkov, Gocho Slavov, Svetoslav Savchev \\ Emails: vgalabov@tu-sofia.bg,_office@spesima.eu, office@spesima.eu, \\ s_savchev@abv.bg
}

\begin{abstract}
The necessity of new approaches for structural synthesis of mechanical systems, which directly identify a limited number of structures that carry a potential for solving the technical problems and meet the specific requirements for the design of mechanical systems, mainly for specialised robots, is specified. The identified five types of kinematic chains (primary, parallel, secondary, additional and subsidiary), with different functionality, allow the synthesis of manipulators structures according to the defined goal tasks and specific functional requirements for hybrid systems, in particular specialised industrial robots. Two mechatronic approaches for functional structural synthesis of mechanical systems of industrial robots, where the main manipulation mechanism is a path generator, were introduced. A limited number of structures that meets the set of objectives and technical requirements to design mechanisms, is directly determined. Emphasis is placed on the tasks of passive (mechanical) control of manipulation systems associated with specialized robotics. Two mechatronic approaches for functional structural synthesis of mechanical systems of industrial robots, where the main manipulation mechanism is a motion generator, are introduced. A limited number of structures is directly defined that meets the set of technical objectives and requirements for the designed mechanisms. Emphasis is placed on the tasks of passive control of the manipulation systems associated with the specialized robotics.
\end{abstract}

Keywords: Structural synthesis, manipulation mechanisms, goal motions, kinematic chains, path-generator mechanisms, motion-generator mechanisms.

\section{Introduction}

The aim of our study is the overall input of new approaches for structural synthesis of mechanisms consistent with the development of the hybrid systems, and more 
particularly of specialised industrial robots. To achieve this goal it is necessary to identify the kinematic chains with different functionality, and on this basis to formulate the basic inter-relationships between the specified coal motion and tasks of the active and passive kinematic control to reach the most rational distribution of the functions of mechanical and control system at an early stage of the design of path-generator mechanisms and motion-generator mechanisms, forming the structure of the mechanical systems of specialized robots.

The basis of the design of robotic manipulators is a functional approach to the structural and dimensional synthesis of mechanisms [3], according to which their structure is built by superimposing kinematic chains with different functionality, which results in control mechanisms [13], which stands are detachable, the basic kinematic chain called primary. These control mechanisms solve the problem of generating the geometry of motion, separating it from the task of active (electronic) control of motion in time.

The main mechanism of the mechanical system of specialized industrial robots is the actuator that performs translations and orientations $[5,6]$. Typically there are three or four degrees of freedom. The structure of the mechanism is usually closedopen to at least one closed-loop of the control transfer mechanism (function generator) $[14,15]$. The introduction of mechanisms with "kinematic intelligence" simplifies the structure and setup of the control system, but requires structural and dimensional synthesis of the mechanism according to the conditions set [3].

Depending on the level of specialization in industrial robots, they are divided into three groups: multi-purpose or universal - intended for various main and auxiliary operations, specialized - designed to perform identical operations for service of process machinery $[2,1,5]$, special - designed for a particular operation or to service a particular machine [22].

The executing mechanisms of universal industrial robots usually have an open kinematic chain with five or six degrees of freedom. Their relatively simple structure and flexible programming are at the expense of a complex structure and setting of the control system [9].

The executing mechanisms of the specialized industrial robots usually have a closed-open kinematic chain with three or four degrees of freedom $[14,6]$. Their structure includes at least one closed-loop control mechanism, generating a part of the goal motion, which simplifies the structure and setup of the control system, but requires structural and dimensional synthesis of the mechanism according to the defined conditions [3].

The executing mechanisms of the special industrial robots are generally composed of one or two modules [25] or have a closed kinematic chain of a control mechanism with one degree of freedom [22]. The control system is maximally simplified at the expense of a complicated synthesis of the control mechanism with a variable transmission rate [3].

In Universal Robotics the control tasks of the manipulators are placed on their design and construction. With the purchase of a suitable universal robot different tasks can be solved, related to the technological and (or) support operations. In most cases, the mechanisms are controlled by an open kinematic chain with an open 
topology, having a sufficient, but in some cases excessive number of degrees of freedom (redundant robots). The intelligent system is relied on for the active control of the task implementation $[8,11]$.

In specialized and special robotics a part of the control tasks of manipulation systems is solved by synthesis of their structure and dimensions for minimum degrees of freedom, required for the realization of the goal task. The generation of movement (moving) trajectory or function, using a mechanism will be summarized conditionally with the term passive kinematic control [2]. This control is assigned to the specially designed control transfer mechanisms [13] that solve the problem of generating the geometry of motion, separating it from the task of controlling the motion in time. In many cases the manipulation or control systems are unnecessarily complicated, if you rely only on the possibility of either a passive or active control.

\section{Development of the structural synthesis}

At the core of the design of mechanical systems lies the synthesis of their mechanisms. The majority of the scientific literature concerning structural synthesis of the mechanisms is directed only towards the task of counting the multitudes of topological schemes of the kinematic structures that do not account for the type of the kinematic pairs, the specific purposes of the synthesis and the functional requirements towards the mechanisms.

By generation of different sets of topological structures and the extraction of the non-isomorphic structures, it is apparent that the development of the structural synthesis of mechanisms is almost at a completed stage [12]. This does not mean that the research work in the field of topological structural synthesis is completed. On the contrary, in papers, such as [25] by $\mathrm{T}$ i s c h 1 e r et al., after a brief review of the existing methods for generating structures and detection of isomorphism more rational possibilities are given to generate a complete list of structures, including a significantly lower number of isomorphic structures. In other works, such as [10] by $\mathrm{C} \mathrm{h}$ e w et al., a conceptual structural synthesis is developed, based on expert systems for certain types of mechanisms. Thereby some of the existing methods are imrpoved, without going beyond the frames of the topological synthesis.

The existing algorithms and programs provide the designer with thousands of structures of mechanisms, but still do not give sufficiently clear orientations for a definitive choice [4]. Therefore, the designer, without precise argumentation and often without a scientific approach selects the structure of the mechanism, which does not always leads to an optimal solution under certain conditions, even when optimzing the mechanism dimension. Instead of using the heterogeneous and lengthy procedure for parallel synthesis (concurrent engineering approach to synthesis), the troubled designer prefers to intuitively focus on a particular structure, in spite of the risk of an unsuccessful choice.

How to avoid the difficulties? Primarily, the duration of the of the mechanical system design must be shortened, without a negative impact on the final product, 
which is most often an innovative project. The largest reserves in this respect can be found at the first stage of the mechanisms design - in their synthesis. New approaches are needed for structural synthesis, by which a limited number of structures can be directly defined, that carry a potential to solve the given technical problem and fulfill the specific requirements of the designed mechanical system.

\subsection{Mechatronic approach for structural synthesis of the mechanisms}

In modern technical devices very close are intertwined the problems of mechanics and electronics of "kinematic" and "electronic" intelligence [20]. The symbiosis of mechanics and electronics leads to rational and cost-efficient technical solutions for various devices [21]. The so-called mechatronic approach has arisen towards the design of mechanisms and manipulators (Mechatronic Design of Mechanisms and Manipulators). This is an approach that gives an appropriate integrated mixture of mechanics, electronics, and software implemented during the design, testing, and production of products and systems to obtain an optimal, under certain conditions, technical solution.

The separability of the function "mechanical motion" in terms of geometry and time was announced by K o n s t a n t i n o v [19] as the first basic principle of mechatronics for decoupling (division) of the motions in space and in time. According to this principle, the task of planning the goal function of "mechanical moton" is separated into a purely geometric part of the overall control task of the mechanical system in space and in time. The mechanisms with a closed structure naturally generate the function "geometry of the motion" in the form of curves of the motion and transfer functions, which are independent on the time parameter but are only determined by the structure and dimensions of their kinematic schemes. When applying input kinematic parameters (speed and acceleration), the motion curves are transformed into trajectories and the transfer functions are converted into kinematic functions because they are now dependent on the parameter time. The synthesis of mechanisms solves the geometric part of the control task of closed kinematic chains [3].

The mechanisms with closed structures have certain advantages over those with open structures ceteris paribus:

- a lower number of degrees of freedom and respectively controllable motors;

- ease of control, since the geometry of complex motions is achieved by the mechanism;

- stabilization of the mechanical system and ability to export drive modules on the frame, which lightens the mobile links;

- trouble-free motion of the end effector, with higher speeds and accelerations on a defined path with improved accuracy and reduced vibrations at the moment of placement.

In order to achieve an increase in the functionality of the mechanical systems, the qualities of the open and closed kinematic chains are combined, which allows to obtain a wide variety of paths in an extended work area. Mixed open-closed kinematic chains are obtained, typical for various specialized robots, including those produced by the Bulgarian-German company SPESIMA. 
Two groups of factors require changes in the approaches to the synthesis of mechanisms. The first one is associated with the synthesis objects. The desire to optimize the internal state and the external interaction between different to their physical nature systems is constant, i.e., hybrid systems. The second group of factors is related to the theoretical apparatus for the synthesis of mechanical systems. Through this apparatus mechanical mathematical models must be drawn related to the specific requirements for the engineered systems and their optimal adaptation.

At home and abroad there has been put a great effort into the theoretical and practical terms for the creation of the so-called intelligent machines. As a first stage in their development the creation of so-called hybrid machines [16] may be adopted, which can be attributed to the specialized robots. Typically, these are devices with two or three degrees of freedom, wherein a corresponding number of servomotors are controlled in such a way, that the output end effector generates a programmable non-uniform motion.

The mechatronic approach for synthesis of mechanisms is developing as a completely new approach towards the design of hybrid technical systems [7]. This approach to synthesis is still in its genesis stage of development, but it is likely to be a realistic and particularly promising direction in the design of hybrid systems [17]. Numerous problems of mechatronics are partially solved or awaiting a solution. In the synthesis of mechanisms for mechatronic devices, such as specialized robots, the priority tasks, without claiming completeness, are:

1. Transformation of the primary target in the specified task assignment for synthesis of the hybrid system made when drafting the plan for its overall design.

2. Preliminary physical and functional decoupling (split) of the target task in space and in time. This is necessary for the synthesis of an optimal structure for a mechanical system which can realize the geometric component of the target motion.

3. Preliminary expert distinction in terms of the features and relative share of the components of mechanics and electronics. This share depends on the actual degree of programmability, required in the implementation of targeted motion, taking into account the reliability of the building blocks of the device, and a number of economic factors.

4. Coordination of the type and location of the mechanical system according to the type and volume of the regional working space, which sets the goal motion. The multitude of kinematically complete kinematic chains in the process of synthesis is reduced to a limited number of competitive options, which completely or partially provides independent (decoupled) position control, which is of significant practical importance.

5. Determination of the optimum number of degrees of freedom of the mechanical system. Limiting their number can be achieved with the introduction of additional connections between the units of the kinematic chains.

6. Multivariate structural synthesis and analysis of a set of possible solutions. This task is directly related to the problem of optimal placement of the 
active (propulsive) kinematic pairs in the boundaries of the whole mechanical system.

7. Choosing a rational method for dimensional synthesis and its implementation, so that the tasks of generating the geometric components of the goal motion to be realized by the mechanical system.

8. Selection of rational methods of force analysis, dynamic, elasto-dynamic synthesis and analysis of the mechanical system. This task is achieved by gradually taking into account the currently formulated priority tasks and is related to the constructive development of the synthesized kinematic schemes.

By solving these problems the development and implementation of some ideas, related to laying the groundwork for the design of mechatronic systems is continued $[15,18,24]$.

\subsection{Findings and conclusions}

From the review in this study and the current status of the theory of structural synthesis of mechanisms, a number of findings and conclusions can be made:

1. The structural synthesis of mechanisms fits poorly into the general theory of control of mechanical systems. The tasks for synthesis of the mechanisms must be considered as the geometric part of the task management of the mechanical systems.

- The question of optimal, under certain conditions, distribution of functions for kinematic and microprocessor control, the motion of the mechanical system remains open. This issue is essentially related to the number of degrees of freedom and structure of the mechanisms.

- The role of the mechanisms in the hybrid systems is not sufficiently clarified, thus the levels of mobility and structure of the mechanisms are unjustifiably determined (mostly closed and with the robots - open) including the type of the kinematic pairs.

- The structural syntehsis is observed as a problem almost isolated from the process of the overall design of the mechanical systems (specifically their dimensional synthesis), and from the contemporary possibilities for control of their motion.

2. At the current stage the structural synthesis is highly developed in terms of a structural formation of lever mechanisms with rotating kinematic pairs (the socalled topological synthesis), and weakly associated with the functional requirements of the mechanisms and the basic tasks of their metric synthesis, which reduces the aggregation of the methods for structural synthesis.

- The structural synthesis is carried out on the basis of closed kinematic chains. The synthesis of mechanical systems with closed-open and open kinematic chains is scantily represented and is developing one-sidedly, mostly for the needs of the universal robotics.

- Many approaches to structural synthesis developed on the basis of graph theory, are not applicable in the general case, mainly due to the lack of necessary and sufficient conditions for the application of these approaches. 
- The choice of the mechanism type is currently insufficiently determinated and thus, even in the most accurate metric synthesis, it rarely leads to an optimal solution under certain conditions.

From the established findings and conclusions the objective of this study is derived in the following three parts, namely to introduce new approaches for structural synthesis with a wide range of applicability, which would facilitate and accelerate the design of modern technical systems. These approaches should be adapted to the maximum extent to the requirements posed by "the symbiosis" of mechanics, electronics and software in the control of defined motions in order to obtain optimal solutions under given conditions.

The study is also oriented indirectly to objectives related to problems for the development of specialized computer programs, as well as possible applications of contemporary technologies in the intelligent design of manipulation mechanisms in specialized robotics [23].

\section{Functional types of kinematic chains}

New approaches are needed for structural synthesis, by which a limited number of structures can be directly assigned, that carry the potential for solving the arising technical problems and to meet the specific requirements towards the designed mechanical system. In this work these approaches have been developed with the idea of building mechanism structures by overlaying open kinematic chains with sequential topology and different functionality [3], forming closed loops of control transfer mechanisms [13]. These unbranched chains are primary building blocks for functional topological synthesis of the manipulation mechanisms. According to their main functionalities, they can be distinguished as primary (prim), parallel (par), secondary (sec), additional (add) and subsidiary (sub).

The primary kinematic chain (Fig. 1, scheme 1 - prim) is the shortest (in number of units) circuit, only with inferior kinematic couples, which connects the end effector (actuator) with the stand. Most universal robots only have a primary circuit, each pair of the kinematic chain being active (driven).

The parallel kinematic chain (Fig. 1, scheme 2 - par) is structurally identical to the primary chain. If there are several parallel chains, then one of them can be considered primary if it contains only lower kinematic pairs. These chains typically create variability of the kinematic configurations of mechanisms and better conditions for their stability, accuracy and balance.

The secondary kinematic chain (Fig. 1, scheme 3 - sec) connects two nonadjacent links of a primary or parallel chain. It is possible that the secondary kinematic chain connects two nonadjacent links of another secondary chain (Fig. 2).

The additional kinematic chain (Fig. 1, scheme 4 - add) connects two nonadjacent links from different kinematic chains (prim, par, sec). Their introduction in the structures is dictated primarily by the need to reduce the degrees of freedom of the mechanism by establishing kinematics dependence on the 
motions of links by two different chains (mostly links associated with the base Fig. 2b, links 1 and 3).

Subsidiary kinematic chain (Fig. 1, a $5-$ sub) connects via two intermediate units two other adjacent units of a primary, secondary or parallel kinematic chain. Typically a four unit topological structure of a mechanism is formed, via which the necessary input motion is set, which if involving downtime, leads to downtime in the subsequent units of the mechanism. Such circuits have typically manipulation mechanisms built into the structures of special robots or machines powered directly by motors without control.

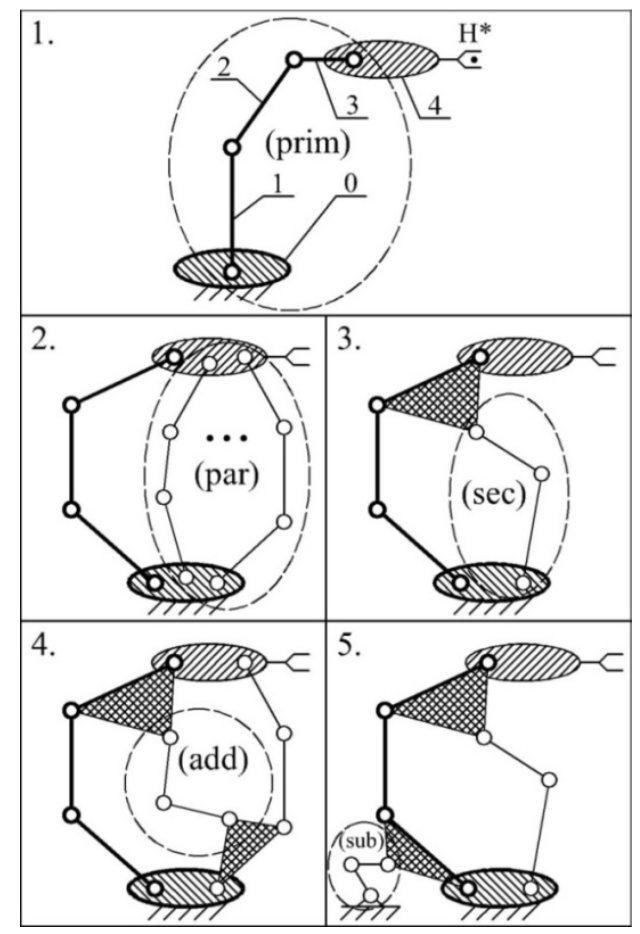

Fig. 1. Functional types of kinematic chains

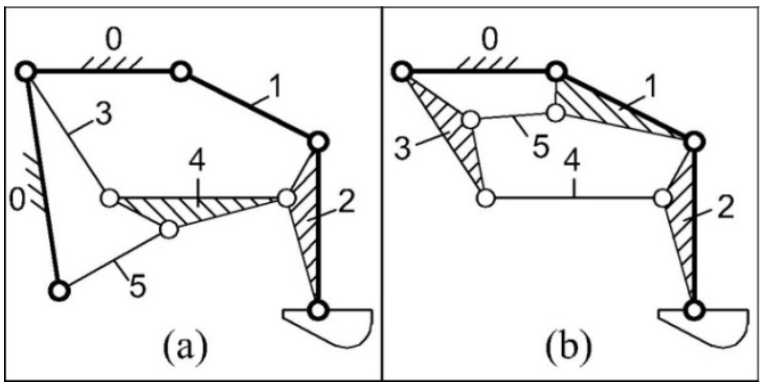

Fig. 2. Functional types of kinematic chains of die casting dosing robots of the firms: (a) Toshiba and Advance: primary - units 0,1,2; two secondary - units 0, 3, 4, 2 и 0, 5, 4 ;

(b) The Bulgarian-German firm SPESIMA (FEEDMAT 1): primary - units 0, 1, 2; secondary - units $0,3,4,2$ and additional $-1,5,3$ 
The secondary and additional kinematic chains are usually included in the creation of closed loops of the so called control transfer mechanisms, to which the relative bases are movable units.

The most simple, in terms of the structure, are the manipulation mechanisms only with a primary chain of the universal robots. The connected kinematic chains normally form closed loops and lower the degrees of freedom and thereby the number of motors, as is the case with the specialized robots. Each attached chain by definition includes also the links, which it connects from one or two other chains. These units are common for associated with them kinematic chains. In this way ternary, quaternary and other links emerge.

The number and character of the functional requirements may be different, because it depends on the concrete task assigned when designing. Eleven examples of functional requirements and their corresponding function types of kinematic chains are given in Table 1. The signs "+" and "-" denote conditionally the functional suitability and functional inadequacy of the five types of kinematic chains to satisfy the requirements.

Table 1

\begin{tabular}{|l|c|c|c|c|c|c|}
\hline \multicolumn{8}{|l|}{ Functional requirements } & \multicolumn{5}{|c|}{ Functional types of kinematic chains } \\
\cline { 2 - 7 } & prim & par & sec & add & sub \\
\hline 1. & $\begin{array}{l}\text { Minimizing the number of the links and the } \\
\text { number of kinematic pairs }\end{array}$ & + & - & - & - & - \\
\hline 2. & $\begin{array}{l}\text { Minimizing the work space of the } \\
\text { mechanism }\end{array}$ & + & - & - & - & - \\
\hline 3. & $\begin{array}{l}\text { Maximizing the work space of the end } \\
\text { effector }\end{array}$ & + & - & - & - & - \\
\hline 4. & $\begin{array}{l}\text { Multivariance of the kinematic } \\
\text { configurations of the mechanism }\end{array}$ & + & + & + & - & - \\
\hline 5. & $\begin{array}{l}\text { Minimizing the number of kinematic pairs, in } \\
\text { which the end effector is present }\end{array}$ & + & - & + & + & - \\
\hline 6. & $\begin{array}{l}\text { Optimizing the number and the order of the } \\
\text { active kinematic pairs }\end{array}$ & - & + & + & + & - \\
\hline 7. & $\begin{array}{l}\text { Minimizing the structural errors of the } \\
\text { mechanism }\end{array}$ & - & + & + & + & - \\
\hline 8. & $\begin{array}{l}\text { Optimizing the actuation via changes in the } \\
\text { order of the active pairs }\end{array}$ & - & - & + & + & + \\
\hline 9. & Stability of the mechanism & - & + & + & + & - \\
\hline 10. & Balancing of the mechanism \\
\hline 11. & $\begin{array}{l}\text { Decoupling of the actuation of the end } \\
\text { effector }\end{array}$ & + & + & + & - & - \\
\hline
\end{tabular}

The goal motion can generally be represented by three components - trajectory $\tau$ of the characteristic point $H$ of the end effector (working instrument or gripper), its speed $V_{H}$ and the angular speed $\omega_{\text {ef }}$ of the effector. This decoupled representation of the motion is appropriate for special robots, as they usually generate unchanging trajectory $\tau$ of the characteristic point $H$ with variable speed $V_{H}$ and with a dependent or independent angular velocity $\omega_{\text {ef }}$ of the effector. 
Each component fits into the summarized concept image or function $(f)$, which is derived from the set theory and belongs $(\in)$ to a set $(F)$ - an area of continuous functions. A function $(f)$ by definition $(::=)$ can be replaced (var) or not replaced (invar) with another function. The field $(F)$ may be defined by condition or defined by the means of one or more related fields $(A, B, \ldots)$, for example $f::=\in \operatorname{var} F \Leftarrow(A, B, \ldots)$.

In this formulation the kinematic components of the goal motion of the end effector, respectively the main tasks for structural synthesis of manipulation systems of industrial robots and for synthesis of their active and (or) passive kinematic control can be formally divided into eight groups (Table 2).

Table 2

\begin{tabular}{|c|c|c|c|c|c|c|c|c|}
\hline Component & $1(\mathrm{~A})$ & $2(\mathrm{~B})$ & 3 & 4 & 5 & 6 & 7 & $8(\mathrm{C})$ \\
\hline$\tau$ & var & invar & var & var & var & invar & invar & invar \\
\hline$V_{H}$ & var & var & invar & var & invar & var & invar & invar \\
\hline$\omega_{\mathrm{ef}}$ & var & var & var & invar & invar & invar & var & invar \\
\hline
\end{tabular}

The most common and typical for the universal robots is task No 1, where the functions of the velocities are independent $\left(V_{H} / \omega_{\text {ef }}::=\right.$ var $)$, so that the minimum number of moving links, degrees of freedom, and required independent input parameters of their open kinematic chain are three. With the specialized robots these functions are generally dependent $\left(V_{H} / \omega_{\text {ef }}:=\right.$ invar $)$, due to the introduction to the primary kinematic chain of another or other functional types of kinematic chains.

\section{Goal motions and structures of path-generator mechanisms}

The goal motion of manipulation path-generator mechanisms in general can be represented by two components - the trajectory $\tau$ of the characteristic point $H$ of the end effector (the gripper or working tool) and its speed $V_{H}$. This decoupled representation of motion is appropriate for specialized robots, as they usually generate unchanging (invar) trajectory $\tau$ of the characteristic point $H$ with a variable (var) speed $V_{H}$.

The kinematic components of goal motion of the end effector, respectively the main tasks for structural synthesis of manipulation systems of industrial robots and for synthesis of their active and (or) passive kinematic control can be formally divided into three groups (Table 3).

Table 3
\begin{tabular}{|c|c|c|c|}
\hline Component & Group A & Group B & Group C \\
\hline$\tau$ & var & invar & invar \\
\hline$V_{H}$ & var & var & invar \\
\hline
\end{tabular}


The tasks of group A are most common for the path-generator mechanisms. The minimum number of moving parts, the number of degrees of freedom and independent input parameters required to open their primary kinematic chain (prim), are 2 (Table 4, A). Then the functions of velocities are conditional $\left(V_{H} / \omega_{\text {ef }}::=\right.$ invar $)$, as in other specialized robots, such as extractors of casts of horizontal machines for high-pressure casting. It is possible the circuit to remain open (Table 4, A) or fully closed (Table 4, B and C). All three groups are common for specialized robots.

To clarify the interconnections between the components of the goal motion, the topological structures, the required input parameters, and consequently the distribution of the tasks of active and passive control, two approaches will be used for closing the primary kinematic chain, through which the topological structures are constructed, presented in Tables 4 and 5.

Column 2 shows the components of the goal motion, column 3 - the corresponding topological structures. The required input parameters are recorded as well (column 4) which must be achieved by active kinematic control of groups A and $\mathrm{B}$. The dependencies in the relative motion (column 5) are necessary for synthesis of the transfer mechanism for passive kinematical control.

\section{First approach to build structures of path-generator mechanisms (Table 4)}

Group A. The trajectory $\tau$ and the velocity $V_{H}$ belong to defined classes of functions defined by sets $T$ and $V: \tau::=\operatorname{var} \in T ; V_{H}::=\operatorname{var} \in V$. The tasks of this group can be solved by mechanisms with a tri-bar open kinematic chain operated by a kinematic system for active control of two generalized coordinates or velocities:

$$
\begin{aligned}
& \dot{\varphi}_{1,0}::=\operatorname{var} \in \dot{\Phi}_{1,0} \Leftarrow(T, V), \\
& \dot{\varphi}_{2,1}::=\operatorname{var} \in \dot{\Phi}_{2,1} \Leftarrow(T, V) .
\end{aligned}
$$

Only one of the two adjustable-speed motors can be directly installed to the frame. The open structure of the mechanism allows the decoupled motion of the two moveable links. This is achieved in the die casting supply robots, such as FEEDMAT 3 of the Bulgarian-German company SPESIMA, where the third link of the prim-chain is the so-called "ladle". The adjustable-speed motors mounted to the frame, move separately the links by gear-chain drives with constant transfer ratios.

Group B. Unlike group A, a change in the goal trajectory is not predicted in the task of this group ( $\tau::=$ invar ). They can be solved by mechanical and control systems, typical for the tasks of group A. A specific solution for the tasks of group B is a part of the motion control of point $H$ of path $\tau$ to be achieved by the mechanism itself.

A parallel kinematic chain (par) with sequential topology is added to the primary kinematic chain. A parallel circuit connects base 0 to link 2 of the primary chain by means of a new link 3 . A control transfer mechanism is formed (a function generator) with a relative base link 1 . The resulting path-generator mechanism is a 
four-bar topological structure with one degree of freedom and respectively an adjustable-speed motor mounted to the frame. The mechanism could in principle generate a trajectory $\tau::=$ invar .

This simplest structure of the class of the so-called Q-manipulators [19], is common for most of the specialized robots, like GRIPMAT extractors of the Bulgarian-German company SPESIMA, which serve to remove the casts of horizontal machines, die casting, and for other purposes.

\begin{tabular}{|c|c|c|c|c|c|}
\hline Group & $\begin{array}{l}\text { Components of } \\
\text { the goal motion }\end{array}$ & $\begin{array}{l}\text { Topologic } \\
\text { structures }\end{array}$ & $\begin{array}{l}\text { Necessary input } \\
\text { parameters }\end{array}$ & \begin{tabular}{|c} 
Dependencies in \\
the related \\
movements
\end{tabular} & $\begin{array}{l}\text { Sample path- } \\
\text { generator } \\
\text { mechanisms }\end{array}$ \\
\hline A & $\begin{array}{c}\tau::=\text { var } \\
V_{H}::=\text { var }\end{array}$ & 1 & $\begin{array}{l}\dot{\varphi}_{1,0}(t)::=\operatorname{var} \\
\dot{\varphi}_{2,1}(t)::=\operatorname{var}\end{array}$ & N/A & \\
\hline B & $\begin{array}{l}\tau::=\text { invar } \\
V_{H}::=\text { var }\end{array}$ & & $\dot{\varphi}_{1,0}(t)::=\operatorname{var}$ & $\begin{array}{c}\varphi_{2,1}=f\left(\varphi_{0,1}\right) \\
i_{2,0}^{(1)}=\frac{\dot{\varphi}_{2,1}(t)}{\dot{\varphi}_{0,1}(t)} \\
\ldots\end{array}$ & \\
\hline $\mathrm{C}$ & $\begin{array}{c}\tau::=\text { invar } \\
V_{H}::=\text { invar }\end{array}$ & & $\begin{array}{c}\dot{\varphi}_{1,0}::=\text { invar } \\
\dot{\varphi}::=\text { const }\end{array}$ & $\begin{array}{c}\varphi_{1,0}=f(\varphi) \\
i=\frac{\dot{\varphi}_{1,0}(t)}{\dot{\varphi}} \\
\varphi_{2,1}=f\left(\varphi_{0,1}\right) \\
i_{2,0}^{(1)}=\frac{\dot{\varphi}_{2,1}(t)}{\dot{\varphi}_{0,1}(t)} \\
\ldots \ldots\end{array}$ & \\
\hline
\end{tabular}

The synthesis of the path-generator mechanism includes sizing of the primary kinematic chain, deriving of the displacement function $\varphi_{2,1}=f\left(\varphi_{0,1}\right)$ and the kinematic control transfer functions $\varphi_{2,1}^{\prime} \equiv d \varphi_{2,1} / d \varphi_{0,1}$ and $\varphi_{2,1}^{\prime \prime} \equiv d^{2} \varphi_{2,1} / d \varphi_{0,1}^{2}$ as a solution to the inverse kinematic problem, synthesis of the control transfer mechanism in these identified transfer functions [6]. The set output speed is achieved through active control of the input speed

$$
\dot{\varphi}_{1,0}::=\operatorname{var} \in \dot{\Phi}_{1,0} \Leftarrow(\tau, V) \text {. }
$$

Group C. In contrast to group B, in this task a change in the function of velocity is not predicted. The tasks of this group can be solved by means of mechanical and control systems, typical for groups A and B. A specific for group C solution can be entirely achieved by means of the mechanical system. The motion 
of point $H$ on curve $\tau$ is performed by the path-generator mechanism, as indicated for the tasks in group $B$. The required function of the generalized velocity

$$
\dot{\varphi}_{1,0}(t)::=\text { invar } \in \dot{\Phi}_{1,0} \Leftarrow\left(\tau, V_{H}\right)
$$

can be generated by means of a subsidiary kinematic chain (sub), which with two intermediate links 4 and 5 kinematically connects base 0 with link 1 (Table 1, C). Two ternary links 0 and 1 are produced and a four-bar closed topological structure of a subsidiary transfer mechanism with an input speed

$$
\dot{\varphi}::=\text { const } \in \dot{\Phi} \Leftarrow\left(\tau, V_{H}\right) \text {. }
$$

The mechanism is synthesized by the function $\varphi_{1,0}=f(\varphi)$ and its derivatives transfer functions, defined by solving the inverse kinematic task with a set law of motion of the outlet $V_{H}=f(t)$ and the linear coordinate $\varphi(t)$ at the input of the mechanism [3]. The overall structure of the mechanism is six-bar of the type Watt II, typical for different types of specialized robots in the recent past, when the control function entirely belonged to the mechanical system. An example of this is the casts extractor of NORDA company (Italy). A subsidiary slot-and-crank mechanism with adjustable length of the node adequately actuates the four-bar main path-generator mechanism, forming with the latter a structure of the type Watt II.

\section{Second approach to build structures of path-generator mechanisms (Table 5)}

Group A. Primary kinematic chain is initially closed by a secondary (sec) kinematic chain which connects to link 2 at point $H$ becoming parallel (par), since it is structurally identical to the primary chain, if it contains only a lower kinematic pair. In this case the structure of a manipulation path-generator mechanism is formed with parallel topology with two degrees of freedom. The tasks of this group are solved by mechanisms with five-bar closed kinematic chain, equipped with a system for active kinematic control of two generalized coordinates or velocities:

$$
\begin{aligned}
& \dot{\varphi}_{1,0}::=\operatorname{var} \in \dot{\Phi}_{1,0} \Leftarrow(T, V), \\
& \dot{\varphi}_{4,0}::=\operatorname{var} \in \dot{\Phi}_{4,0} \Leftarrow(T, V) .
\end{aligned}
$$

Group B. One additional kinematic chain (add) is superimposed on the resultant structure (group A). The chain includes links 1 and 4, associated with a new (intermediate) link 5. Links 1 and 4 are ternary. A four-bar loop for the control transfer mechanism with base 0 is formed, that generates geometric dependencies between the relative movements of links 1 and 4 for passive control of the geometric components of the goal motion of point $H$.

The additional introduction of binary link 5 reduces the degrees of freedom by 1. The resulting six-bar manipulation mechanism is of Stephenson I type, as is the structure of die casting robots FEEDMAT 1 of the Bulgarian-German company SPESIMA, characterized by high energy efficiency compared to the die casting robots of other companies.

Group C. In order to control the kinematic components of the goal motion, a subsidiary kinematic chain (sub) can be brought, which connects base 0 to link 1 
through links 6 and 7 (Table 5, C). Thus the base link 0 becomes ternary and link 1 - quaternary. Finally a subsidiary transfer mechanism is formed with a four-bar topological structure (usually with a high kinematic pair), which generates the law of motion $\varphi_{1,0}=f(t)$ of link 1 , set by solving an inverse kinematic problem. In the past two decades, this law is generated directly by controlling the parameters of the electric motors, which leads to simpler structures of the manipulators of groups B and $\mathrm{C}$ in Tables 4 and 5.

\begin{tabular}{|c|c|c|c|c|c|}
\hline Group & $\begin{array}{l}\text { Components of } \\
\text { the goal motion }\end{array}$ & $\begin{array}{l}\text { Topologic } \\
\text { structures }\end{array}$ & $\begin{array}{l}\text { Necessary input } \\
\text { parameters }\end{array}$ & $\begin{array}{c}\text { Dependencies in } \\
\text { the related } \\
\text { movements }\end{array}$ & $\begin{array}{l}\text { Sample path- } \\
\text { generator } \\
\text { mechanisms }\end{array}$ \\
\hline A & $\begin{array}{c}\tau::=\operatorname{var} \\
V_{H}::=\text { var }\end{array}$ & & $\begin{array}{l}\dot{\varphi}_{1,0}(t)::=\operatorname{var} \\
\dot{\varphi}_{4,0}(t)::=\operatorname{var}\end{array}$ & N/A & \\
\hline B & $\begin{array}{l}\tau::=\text { invar } \\
V_{H}::=\text { var }\end{array}$ & & $\dot{\varphi}_{1,0}(t)::=\operatorname{var}$ & $\begin{aligned} \varphi_{4,0} & =f\left(\varphi_{1,0}\right) \\
i_{4,1}^{(0)} & =\frac{\dot{\varphi}_{4,0}(t)}{\dot{\varphi}_{1,0}(t)}\end{aligned}$ & \\
\hline $\mathrm{C}$ & $\begin{array}{c}\tau::=\text { invar } \\
V_{H}::=\text { invar }\end{array}$ & 14 & $\begin{array}{c}\dot{\varphi}_{1,0}::=\text { invar } \\
\dot{\varphi}::=\text { const }\end{array}$ & $\begin{array}{c}\varphi_{1,0}=f(\varphi) \\
i=\frac{\dot{\varphi}_{1,0}(t)}{\dot{\varphi}} \\
\varphi_{4,0}=f\left(\varphi_{0,1}\right) \\
i_{4,1}^{(0)}=\frac{\dot{\varphi}_{4,0}(t)}{\dot{\varphi}_{1,0}(t)} \\
\ldots \ldots \ldots\end{array}$ & \\
\hline
\end{tabular}

\section{Goal motions and structures of motion-generator mechanisms}

The goal motion consists of three components - a movement curve $\tau$ of the characteristic point $H$ of the end effector (a gripper or working instrument), its velocity $V_{H}$, and angular velocity $\omega_{\text {ef }}$ of the effector.

In this formulation the kinematic components of the goal motion of the end effector, respectively the main tasks for structural synthesis of manipulation systems of industrial robots and for synthesis of their active and (or) passive kinematic control were formally divided into eight groups. The manipulation pathgenerator mechanisms groups are three (A, B and C), since they do not take into 
account the angular velocity $\omega_{\text {ef }}$ of the effector. Accordingly, the components of the goal motion in this section of the manipulation motion-generator mechanisms will be again classified into three groups, but will also include subgroups, since the angular velocity $\omega_{\mathrm{ef}}$ of the effector is taken into account and they are shown in Table 6.

Table 6
\begin{tabular}{|c|c|c|c|}
\hline Component & Group A & Group B & Group C \\
\hline$\tau$ & var & invar & invar \\
\hline$V_{H}$ & var & var & invar \\
\hline$\omega_{\text {ef }}$ & var & var & invar \\
\hline
\end{tabular}

Again the approaches for structural development of the manipulation mechanisms are two.

First approach to build structures of motion-generator mechanisms (Table 7)

Group A. The trajectory $\tau$, velocities $V_{H}$ and $\omega_{e f} \equiv \omega_{3,0} \equiv \dot{\varphi}_{3,0}$ belong to preset classes of functions, defined by the sets $T, \quad V$ and $\Omega: \tau::=\operatorname{var} \in T$; $V_{H}::=\operatorname{var} \in V, . \omega_{3,0}::=\operatorname{var} \in \Omega_{3,0}$. This goal task for generating a programmable changable trajectory and orientation is most common and characteristic for the universal robots, where the functions of the velocities are independent $\left(V_{H} / \omega_{e f}:=\right.$ var $)$, due to which the minimum number of moveable links, degrees of freedom, required input parameters of their open kinematic chain and respective manageble engines is 3 . This task can be solved by mechanisms with a four-link open primary kinematic chain, guided by a system for active kinematic control of three generalized coordinates or velocities:

$$
\begin{aligned}
& \dot{\varphi}_{1,0}(t)::=\operatorname{var} \in \dot{\Phi}_{1,0} \Leftarrow\left(T, V, \Omega_{3,0}\right), \\
& \dot{\varphi}_{2,1}(t)::=\operatorname{var} \in \dot{\Phi}_{2,1} \Leftarrow\left(T, V, \Omega_{3,0}\right), \\
& \dot{\varphi}_{3,2}(t)::=\operatorname{var} \in \dot{\Phi}_{3,2} \Leftarrow\left(T, V, \Omega_{3,0}\right) .
\end{aligned}
$$

The open structure of the mechanism allows the decoupled propulsion of the three moveable links. This is achieved by the die casting supply robots of FEEDMAT 3 type of the Bulgarian-German firm SPESIMA, where the third moveable link is the ladle itself, which scoops and doses the melted aluminum alloy.

Group B. In contrast to group A, in the tasks of these groups a change of the target trajectory is not expected ( $\tau::=$ invar). They can be solved with mechanical and control systems, typical for the tasks of group A. A specific for the tasks from group $B$ solution is a part of the control of the motion of point $H$ along the curve $\tau$ fulfilled by the mechanism itself.

Subgroup B1. One secondary kinematic chain (sec1) is formed by base 0 , link 2 of the primary chain and one new intermediate link 4. A new control four-bar linkage is formed with a relative base link 1 . The reverse kinematic task is solved, 
in which correlations between the relative motions of links 0,1 and 2 in the shape of a displacement function $\varphi_{2,1}=f\left(\varphi_{0,1}\right)$ are derived and the kinematic transfer functions $i_{2,0}^{(1)}, i_{2,0}^{\prime(1)}, \ldots$, representing consecutive derivatives of the displacement functions $\varphi_{2,1}$ related to $\varphi_{0,1}$. With the help of these functions the aforementioned control transfer mechanism for passive kinematic control is synthesised. In reality trajectory $\tau$ is generated by the formed four-bar path-generator linkage, typical for many of the specialized robots of GRIPMAT type, which are designed to extract casts of horizontal machines for pressure casting, as well as for other purposes. The fourth, also separately moved link, is a translational module, equipped with a gripper.

The generalized velocities (1) and (2) must be synchronized in such a way, that at defined permanent geometric parameters of the motion-generator mechanism to obtain the set components $V_{H}$ and $\omega_{3,0}$ of the goal motion.

Subgroup B2. A secondary kinematic chain (sec2) is formed by links 1, 3 of the primary chain and one new intermediate link 5. A four-bar path-generator linkage is formed with a relative stand link 2 . The reverse kinematic problem is solved, where correlations between the relative motions of links 1,2 and 3 in the shape of the displacement function $\varphi_{3,2}=f\left(\varphi_{1,2}\right)$ are derived and the kinematic transfer functions $i_{3,1}^{(2)}, i_{3,1}^{\prime(2)}, \ldots$, representing consecutive derivatives of the displacement function $\varphi_{3,2}$ in relation to $\varphi_{1,2}$. With the aid of these functions the aforementioned motion-generator mechanism for passive kinematic control is synthesized.

This structure $\mathrm{B} 2$ is identical to $\mathrm{B} 1$ under the condition, that base 0 and the end-link 3 are inversed. Regardless of this, the motion-generator mechanism with structure B2 cannot fulfill the goal motion of a mechanism with structure B1, in particular the condition $\omega_{3,0}::=\operatorname{var} \in \Omega_{3,0}$. The function $\omega_{3,0}$ is dependent on the functions $\omega_{1,0} \equiv \dot{\varphi}_{1,0}, \quad \omega_{2,1} \equiv \dot{\varphi}_{2,1} \quad$ and the dimensions of the motion-generator mechanism with a relative stand 2 , input link 1 and output link 3 . By changing $V_{H}$ $\omega_{1,0}$ and $\omega_{2,1}$ are changed. Therefore $\omega_{3,0}$ cannot be independently controlled.

Subcategory B3. The trajectory $\tau$ is generated by a path-generator mechanism, typical for B1, while the orientation of the end effector (unit 3) is achieved by a second control transfer mechanism, typical for group B2. In practice the structure of subgroup B3 can be observed as a combination of the subgroups B1 and B2, derived by overlaying a primary kinematic chain with two secondary chains, where a structure of the type Watt II is formed, which is a particular case of the so-called $Q$-manipulators [19], among which is SPEEDMAT robot of the Bulgarian-German firm SPESIMA. The actively controled generalized velocity $\omega_{1,0} \equiv \dot{\varphi}_{1,0}$ of the mechanism, represented by indication (1), is derived as a solution 
of the reverse kinematic chain under certain permanent parameters of the kinematic scheme of the mechanism and a given function of the output velocity $V_{H}$.

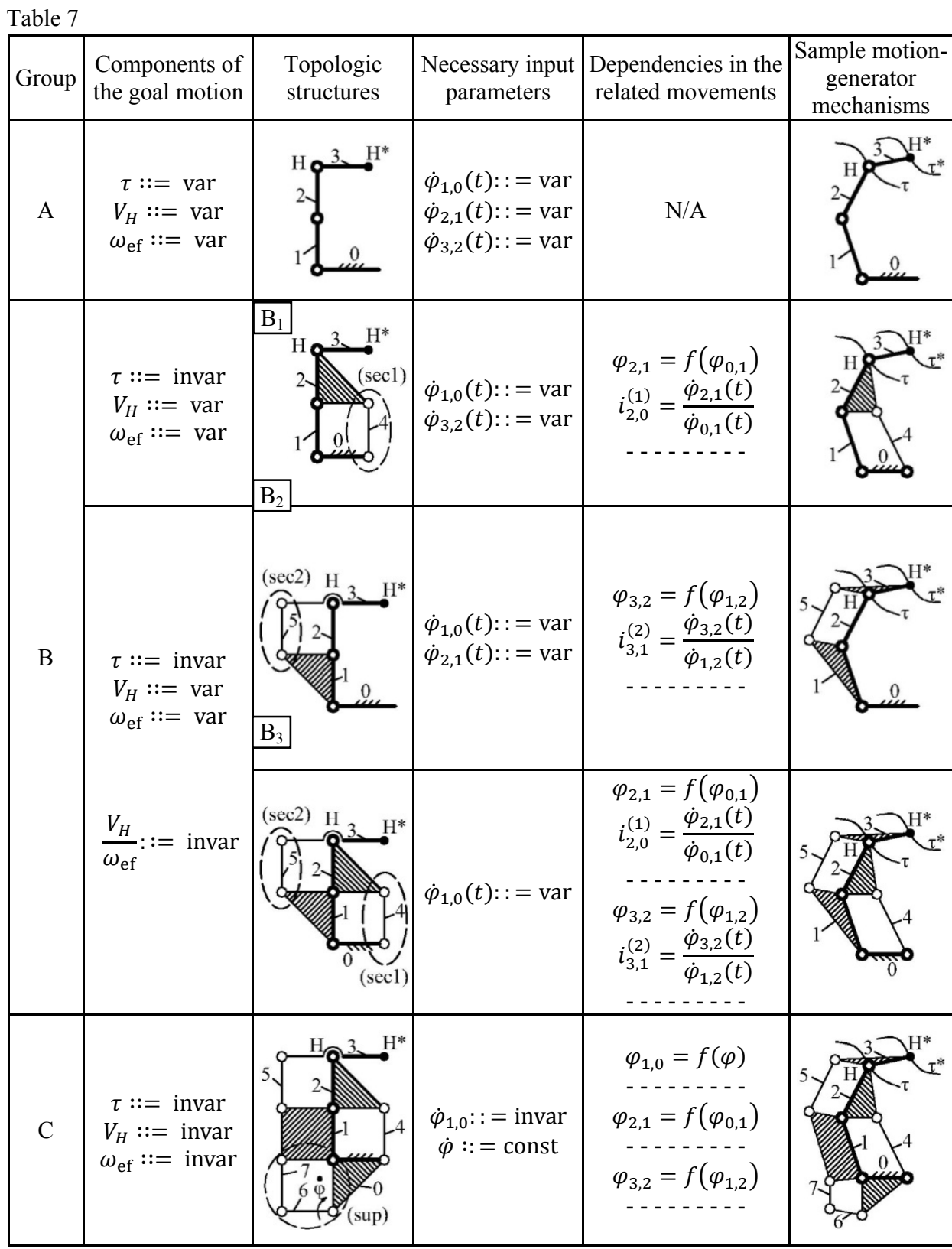

Group C. In contrast to the subgroups of group B in the tasks of group C, a change of the functions, defining respectively the velocity of point $H\left(V_{H}::=\right.$ invar $\left.\in \mathrm{V}\right)$ and the angular velocity of the end effector $\left(\omega_{3,0}::=\right.$ invar $\left.\in \Omega_{3,0}\right)$ is not expected. The tasks of this group can be solved with 
the help of mechanical and control systems, typical for groups A and B. Specifically for group $\mathrm{C}$, a solution is achieved entirely with the help of the mechanical system. The movement of point $H$ along curve $\tau$ is realized by the path-generator mechanism, as described by the tasks of group B. The required function of the generalized velocity

$$
\dot{\varphi}_{1,0}(t)::=\operatorname{invar} \in \dot{\Phi}_{1,0} \Leftarrow\left(\tau, V_{H}\right), \omega_{3,0}
$$

At $\tau::=$ invar $\in T ; V_{H}::=$ invar $\in V, \omega_{3,0}::=$ invar $\in \Omega_{3,0}$, will be derived by means of the subsidiary kinematic chain (sub), which by two binary links 6 and 7 connects kinematically base link 0 with link 1 . A four-bar topolotical structure is formed of a subsidiary transfer mechanism with input velocity

$$
\dot{\varphi}::=\text { const } \in \dot{\Phi} \Leftarrow\left(\tau, V_{H}, \omega_{3,0}\right) .
$$

The mechanism is synthesized with the function $\varphi_{1,0}=f(\varphi)$ and its derivative transfer functions, defined by solving a reverse kinematic chain at set law of motion of the output $V_{H}=f(t)$ and linear coordinate $\varphi(t)$ at the entry of the mechanism [6]. The overall structure of the mechanism is of an eight-bar type of the class of the $Q$-manipulators.

\section{Second approach to build structures of motion-generator mechanisms (Table 8)}

Similar to the first approach, a structure of a manipulation mechanism with three degrees of freedom is observed, after which the levels of mobility are reduced, by inputting other functional types of chains, where four-bar loops of control transfer mechanisms for passive control are derived.

Group A. It is comprised of three subgroups, presented in Table 8, where the components for the goal motion, the topological structures, the necessary input parameters, the dependencies between the relative motions and sample motiongenerator mechanisms are provided (as in Table 4). However, in contrast to the ones in Table 1, higher kinematic pairs are included.

Subgroup A1. A four-bar primary kinematic chain is initially closed by a parallel one (par). The structure of a manipulation mechanism with a parallel topology and three degrees of freedom is formed. Two of the three adjustable-speed motors are to be appropriately mounted on the frame.

Subgroup A2. One additional kinematic chain (add1) is formed by links 1 and 5 , connected to base 0 , and one new intermediary binary link 6 , with which the number of the degrees of freedom is reduced to two. This structure does not allow only one of the two adjustable-speed motors to be mounted on the frame. This opportunity allows the next subgroup.

Subgroup A3. One additional kinematic (add2) is formed by links 2 and 4, connected to the output link 3 , and one new intermediate binary link 7 . The number of the degrees of freedom remains two. The advantage of the structure A3 in comparison to $\mathrm{A} 2$ is the opportunity both motors to be mounted on the frame. 


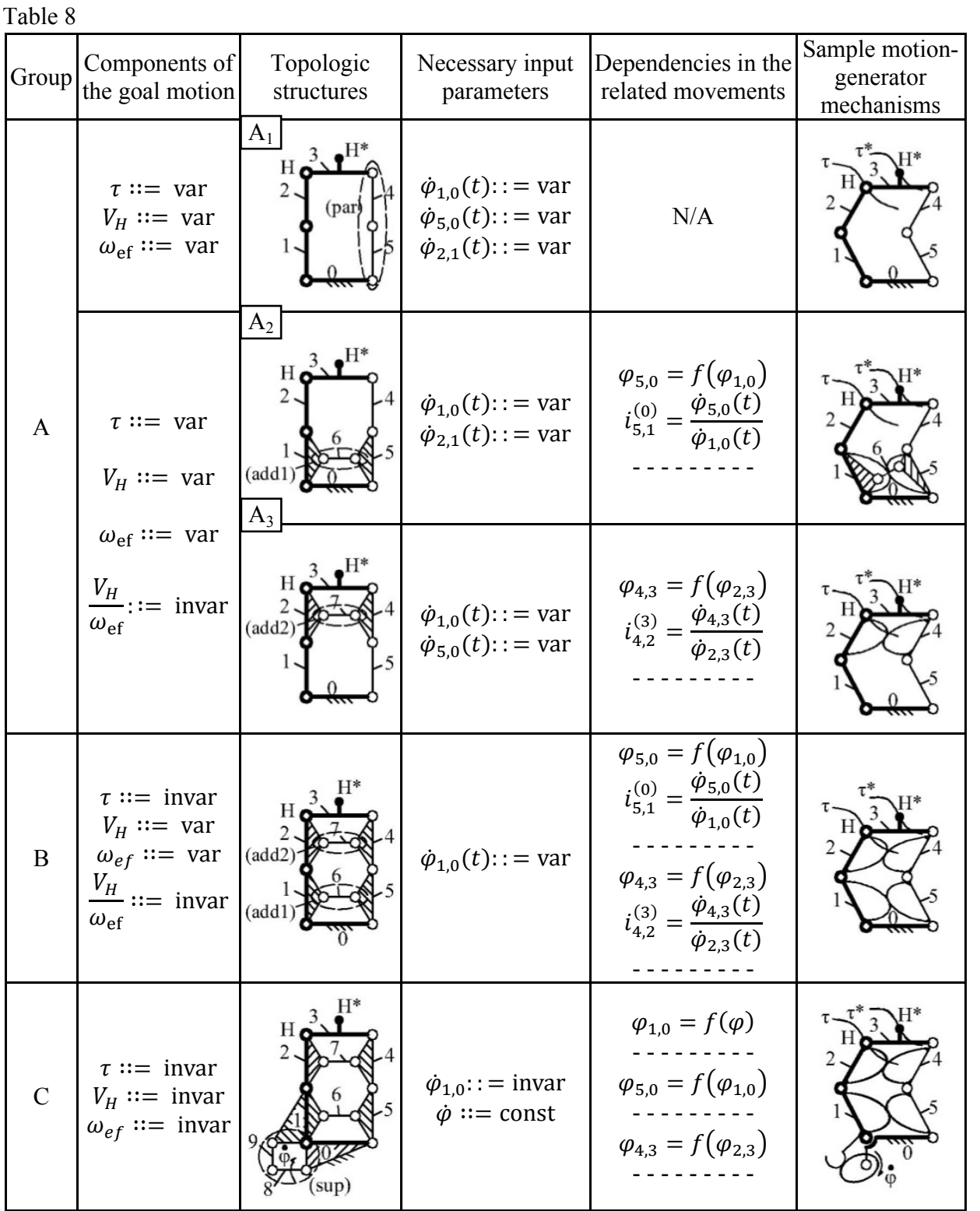

Group B. The structure of this subgroup can be observed as a combination of subgroups A2 and A3, attained after entering two additional kinematic chains (add1 and add2). An eight-bar manipulation mechanism is obtained with one degree of freedom and respectively one adjustable-speed motor, which can appropriately be mounted on the frame.

Group C. The structure of this group can be formed by the previous subgroup A3 after inputting the subsidiary kinematic chain (sub), which with two binary links 8 and 9 connects kinematically base link 0 with link 1. A four-bar topological structure of an subsidiary motion-generator mechanism with input velocity (5) is formed. 


\section{Conclusion}

In Section 2 of this study the necessity for new approaches to structural synthesis of mechanical systems is discussed. In Section 3 five types of kinematic chains with different functionalities are identified, as well as goal tasks and functional requirements for manipulation mechanisms are formulated, consistent with the control of industrial robots. On this basis, in Section 4 two mechatronic approaches for functional structural synthesis of mechanical systems of industrial robots are introduced, where the main manipulation mechanism is a path generator. Similarly to those of Section 5, both mechatronic approaches are developed and oriented towards more complex manipulation mechanisms - motion generators. These approaches make possible to directly identify a limited number of potential opportunities to solve the occurring technical problems and to meet the specific requirements of designing primarily mechanisms of specialized robots.

Emphasis is placed on the tasks of passive control of the manipulation systems associated with specialized robotics. These problems are solved by the functional synthesis of their structure and dimensions at a minimum number of degrees of freedom, required for the realization of the goal task.

\section{References}

1. A v r a mov, I., V. G a $1 \mathrm{a} b$ o v, N. N i k o 1 o v. Applications of the Mechatronic-Adequate Approach in the Study and Design of Robots, Transferring Liquids. - Technical University of Sofia, Annual Edition, Vol. 50, 1999, No 3, 94-101 (in Bulgarian).

2. G a $1 \mathrm{a}$ b o v, V., V. M i h a i 1 o v, I. A v r a m o v. Mechatronic Specification of the Basic Problems of Synthesis and Kinematic Control of Mechanisms in Special Robotics. Machines Mechanics, 1996, No 14, 89-101 (in Bulgarian).

3. G a 1 a b o v, V. Structural-Metric Synthesis of Mechanisms. Ph. D. Thesis, Technical University of Sofia, 1998. 491 p. (in Bulgarian).

4. P e j s a h, E. E. Structural Synthesis of Flat Sock Mechanisms: Contemporary State and Actual Problems. - Machines Mechanics, 2008, No 75, 87-93 (in Russian).

5. S l a v k o v, V. N. N i k o l o v, V. G a l a b o v. Functional Typology and Structural Classification of Moulder Dosing Robots. - Machines Mechanics, 2004, No 50, 64-71 (in Bulgarian).

6. Slavkov, V., V. Galabov. Mechatronic Approach towards the Study, Design and Development of a Specialized Robot. - Automatics and Informatics, 2005, No 4, 46-50 (in Bulgarian).

7. A 11 e n, R. G. Mechatronics Engineering: A Critical Need for This Interdisciplinary Approach to Engineering Education. - In: Proceedings of 2006 IJME-INTERTECH Conference, ENG 205-085.

http://www.ijme.us/cd_06/PDF/ENG\%20205-085.pdf access 12.02.2012

8. A v r a m o v, I., V. M i h a i 10 v, V. G a l a b o v. Computer Aided Design of Mechanisms Based on Some Mechatronics Ideas. - In: Mechatronics - The Basis for Now Industrial Development. M. Acar, Ed. Joint Hungarian-British Mechatronics Conference, Budapest, Computational Mechanics Publications, 1994, 451-460.

9. B a 1 a v e s s o v, V., V. G a 1 a b o v, I. A v r a mov, Y. D e y a n o v. Adaptive Motion Control of Mechatronic Systems. - In: Proceedings of 2nd International Conference on Recent Advances Mechatronics ICRAM'99, 24-26 May, Istanbul, 1999, 153-158.

10. C h e w, M., S. N. T. C h e n, , G. F. Issa. Kinematic Structural Synthesis of Mechanisms Using Knowledge- ased Systems. - J. of Mechanical Design, Vol. 117, March 1995, 96-103. 
11. D i m i t r o v, L., T. N e s h k o v. Intelligent Manufacturing Systems and Mechatronics, An Educational Approach. - In: 1st International Scientific Conference on Engineering Manufacturing and Advanced Technologies MATT'2010, Mostar, Bosnia and Herzegovina, 18-20 November 2010, 117-122. ISSN 1986-9126.

12. Erdman, A. G., Ed. Modern Kinematics. New York, John-Wiley \& Sons, Inc., 1993, 604 p.

13. G a 1 a b o v, V. Structure, Synthesis and Application of Q-manipulators. - In: Proceedings of 6th World Congress on the TMM, Vol. 2, 1983, 1007-1010.

14. G a 1 a b o v, V., I. A v r a m o v, V. M i c h a i 1 o v, Z. S o t i r o v, J. D e a n o v. TypeDimensional Synthesis of Mechanisms for Robot Manipulators. - In: Proceedings of 6th International Machine Design and Production Conference (UMTIC'94), Ankara, 1994, 159-170.

15. G a 1 a b o v, V., I. A v r a m o v, V. M i c h a i 1 o v, R. B o t e v. Concurrent Formulation of Basic Problems in Synthesis and Control of Manipulating Mechanisms Used in Task-Specific Robots. - In: Proceedings of 2nd ECPD International Conference on Advanced Robotics, Intelligent Automation and Active Systems, Vol. 1, Wienna, 1996, 247-251.

16. G r e e n o u g h, J. D., et al. Design of Hibrid Machines. - In: Proceedings of the IX Congress of TMM, Milano, Vol. 4, 1995, 2501-2505.

17. $\mathrm{H}$ a b i b, M. Interdisciplinary Mechatronics Engineering and Science: Problem-Solving, CreativeThinking and Concurrent Design Synergy. - International Journal of Mechatronics and Manufacturing Systems, Vol. 1, 2008, No 1, 4-22.

18. I s e $\mathrm{r} m$ a $\mathrm{n}$, R. On the Design and Control of Mechatronic Systems - A Survey. - IEEE Transactions of Industrial Electronics, Vol. 43, 1996, No 1, 4-15.

19. K o n s t a n t i n o v, M. First Principle of Mechatronics: Motion Distribution with Respect to the Space and Time. - In: Proceedings IFTOMM Symposium, Bulgaria, 1987.

20. K o n s t a $\mathrm{n} \mathrm{t}$ i n o v, M. Mechatronics in Robotics. - In: Proceedings of 7th CISM-IFTOMM Symposium on Theory and Practice of Robots and Manipulators, RoManSy 7, Paris, Hermes, 1990, 252-263.

21. L e n g e r k e, O., S. D u t r a, M. T a v e r a. Mechatronics Education - Synergistic Integration of New Paradigm for Engineering Education. - In: Proceedings of 6th National Congress of Mechanical Engineering, 18-21 August 2010. Campina Grande, Paraíba, Brazil (access 07.02.2012).

http://www.omarlengerke.com/mechatronics\%20definition\%20Lengerke.pdf

22. L u c k, K., K.-H. M o d l e r. Synthesis of Guidance Mecahnisms. - Mecanism and Machining Theory, Vol. 29, 1994, No 4, 525-533.

23. M i c h a i $1 \mathrm{o} \mathrm{v}$, V., V. G a 1 a b o v, I. A v r a m o v. Software Tools for CAD of Mechanism Development and Application in Mechatronic Environment. - Engineering Mechanics, Assoc. for Eng. Mechanics, Vol. 3, 1996, Brno, Czech. Republic, No 2, 97-102.

24. P o p, G., V. M a t i e s. Considerations about the Mechatronical Transdisciplinary Knowledge Paradigm. - In: Proceedings of IEEE International Conference on Mechatronics, Malaga, Spain, 14-17 April 2009, 1-4.

25. T i s c h l e r, C. R., A. E. S a m u e 1, H. K. H u n t. Kinematic Chains for Robot Hands. I. Orderly Number Synthesis. II. Kinematics Constrains, Classification, Connectivity and Actuation. Mech. Mach. Theory, Vol. 30, 1995, No 8, 1193-1239. 